\title{
Health promotion indicators: current status, issues and problems
}

\author{
DAVID MCQUEEN \\ Research Unit in Health and Behavioural Change, University of Edinburgh, United Kingdom
}

HORST NOACK

Department of Social and Preventive Medicine, University of Berne, Switzerland

\section{SUMMARY}

This article describes the background to the current status of health promotion indicator research and defines the major issues and problems involved. It then discusses both of these areas in detail. Problems in health promotion indicators fall under three major headings: methodologies, concepts and theories, and purpose and applications. Two other kinds of problems are discovered under these headings. Problems of methodology include difficulties in measurement and data analysis. The issues in concepts and theories of indicators include defining the field and defining the theory, while issues in purposes and applications involve the use of indicators in the fields of research and policy. The authors conclude that the emerging paradigm of health promotion has a wider perspective than the paradigm of disease prevention, medical care and medical treatment.

\section{INTRODUCTION}

During the 1980 s, as a result of growing discussion of and concern over the inadequacy of health education and prevention of disease, the search for adequate indicators for health promotion intensified. This discussion led to the consideration of new indicators to measure changes in positive health and to evaluate activities and programmes aiming at better health for the population.

In general, the discussion has been informed by the targets of the World Health Organization (WHO) strategy for health for all (WHO, 1985) and the concept and principles of health promotion (WHO, 1984).

More specifically, several key efforts may be noted: an editorial board, convened in 1981 by the International Epidemiological Association and the WHO Regional Office for Europe began to work on a book, Measurement in health promotion and protection (Abelin et al., 1987). A parallel effort began in February 1986, in Edinburgh, following the Symposium on Health Behaviour: Its Application to Health Promotion (Anderson et al., in press). The Symposium, in Pitlochry, Scotland, was held to initiate a process of identifying health promotion indicators. In addition to a preliminary review and discussion of the current state of indicators relevant to health promotion, the Edinburgh working group outlined the agenda for the Workshop on Measuring Health Behaviour and Health: towards New Health Promotion Indicators, held in Berne, in June 1986, papers for which form the basis of this special issue of Health promotion. Following the Berne Workshop a special session on indicators was held at the first International Conference on Health Promotion in Ottawa. Thus the discussion of indicators was placed clearly on the agenda of health promotion. In this session, the official report on the Berne Workshop (WHO, 
1986) was presented. Further, the Ottawa Charter for Health Promotion (1986) came to be regarded as an additional primary document in the development of new health promotion indicators.

Against this background a consultation on indicators was held in Edinburgh in January 1987. Eight consultants from Denmark, France, Switzerland and the United Kingdom met for two days to consider general issues and, more particularly, the WHO targets for health for all concerned with lifestyle, and the indicators for health promotion. In August 1987, at the International Conference on an Intercantonal Health Indicators Study in Switzerland in Lausanne, some time was devoted to indicators for health promotion and disease prevention. Finally, in October 1987 a meeting in Edinburgh considered indicators in the context of the new public health and its implications for research.

During this intensive and sustained process, the Berne Workshop represented a critical step in the discussion of health promotion indicators, principally because most of the key issues and problems in indicator development became manifest. The same issues and problems have continually re-emerged at each stage of this process.

\section{PROBLEMS AND ISSUES}

These emergent problems and issues might be classified in a number of ways. For example, they could be classified according to their methodology, insolubility, expediency, saliency, or usefulness in the strategy for health promotion. These are just a few examples. The classifications offered here (Table 1 ) represent the views of the authors after consideration of a number of alternatives.

\section{Methodologies}

The search for health promotion indicators essentially began with methodological issues, classical questions of operationalization, data collection and data analysis. It was only after difficulties in deciding what to measure, why, and what for, that the emphasis began to shift towards concepts and application.

To begin with, there was the hope or belief that traditional indicators and measures of disease and health could be easily transformed or would suffice for health promotion. Therefore early discussions concentrated heavily on reviews of measures of health status. The principal methodological problem with these classical measures is their validity for measuring health or state of wellbeing. They are chiefly concerned with deviations from normal physiological and social functioning. That is, they tend to concentrate on disability. The article by Kaplan is an excellent example of a well developed methodological approach to measuring such departures from normality. Such measures may be valid and reliable for the measurement of morbidity and mortality, but not of health. This validity issue has remained a constant theme throughout all the discussions on indicators of

Table 1: Issues and problems in health promotion indicators

\begin{tabular}{|c|c|c|}
\hline Purpose and applications & Concepts and theories & Methodologies \\
\hline Research & Defining the field & Measurement \\
\hline $\begin{array}{l}\text { Testing of theory } \\
\text { Prediction of change }\end{array}$ & $\begin{array}{l}\text { Universality versus specificity } \\
\text { Meanings of health promotion } \\
\text { indicators }\end{array}$ & $\begin{array}{l}\text { Data source } \\
\text { Qualitative versus quantitative } \\
\text { data }\end{array}$ \\
\hline Explanation or understanding & $\begin{array}{l}\text { Subjective versus objective } \\
\text { indicators }\end{array}$ & Reliability and validity of data \\
\hline Development of new techniques & $\begin{array}{l}\text { Static versus dynamic indicators, } \\
\text { context of indicators }\end{array}$ & Data collection \\
\hline Polley & Defining the theory & Data analysls \\
\hline $\begin{array}{l}\text { Baseline information } \\
\text { Monitoring change } \\
\text { Evaluation of interventions }\end{array}$ & $\begin{array}{l}\text { Micro- versus macro-level } \\
\text { Process versus input, output } \\
\text { models }\end{array}$ & $\begin{array}{l}\text { Simple versus composite } \\
\text { Statistical versus mathematical } \\
\text { Presentation }\end{array}$ \\
\hline
\end{tabular}


health promotion. From the viewpoint of psychometric measurement this is a problem in construct validity. The underlying construct, health, is not being measured.

\section{Measurement}

This issue leads to discussions of the operationalization of health as a construct. Hunt makes the point in her article that scientists tend to assume that the experience of health must be measurable in objective terms in order to be "scientific". They attempt to measure what is in essence a subjective state by an objective strategy. This fundamental epistemological issue has simply not been as fully comprehended as it might have been.

A related issue is the assumption that levels of wellbeing can be distinguished or measured in the same way as levels of ill health. Hunt believes this is net possible because people experience wellbeing as an emotional phenomenon that is "holistic" in individuals. Thus methods that artificially split this phenomenon into parts will not validly measure the essential nature of wellbeing. That is, people tend not to divide their wellbeing into functional parts, some of which are emotional and some social.

The result of this discussion was the general consensus that health must be viewed both objectively and subjectively. This led to discussions of indicators that might include both objective and subjective components. The methodological problem then is how to combine such components. To date, there is no readily acceptable method of doing this.

A related issue is that of complex versus simple measures of indicators of health and health promotion. On the one hand, there is a strong feeling that the simple measure of asking people how they rate their health or wellbeing (excellent, good, fair or poor) is a satisfactory solution to the operationalization of health. Indeed, it is a global measure that requires respondents to express "holistically" their feeling of wellbeing. Further, it has been shown in several studies to be a valid predictor of morbidity and so-called illness behaviours such as the use of primary and secondary health services. On the other hand, the measure may be unstable over time and depend upon a variety of contextual factors. For example, as people's expectations for excellent health rise over time, owing to contextual factors that may stem from increasing expectations of medical solutions to individual and social problems, individuals may perceive a discrepancy between their expectations and actual situation. This leads over time to a decline in overall perceived level of wellbeing as measured by this simple item.

This change in individual expectations relates to the issue of the individual as the only source of data for health promotion indicators. If health is regarded only as the responsibility of the individual, then it becomes methodologically difficult to incorporate in its measurement the contextual factors that have now been recognized as influencing the global perception of wellbeing. Data collection for traditional indicators of both health and illness has relied almost exclusively on self-reports or medical procedures performed on individuals.

Nevertheless, the contextual problem is not solved by assessing available data on individuals. Groothoff's article is a good illustration. Although it appears relatively straightforward to measure years lost from work due to disability, Groothoff documents the paradox that, while years lost are rapidly increasing in the population, the general mortality rate is decreasing and life expectancy growing. Thus, one so-called objective, hard indicator seems to attest to declining health status while the so-called most objective indicator, mortality, is improving. It is clear that contextual factors such as powerful changes in the social welfare system (in such areas as work) have changed the meaning of the so-called objective measure in one country, the Netherlands.

Data sources for health promotion indicators that could incorporate contextual effects have not been systematically considered. Examples of such sources are measures of: social support networks (as discussed in Mootz's article); the sociocultural environment and effects on healthrelated behaviour (as discussed in the article by Bucher \& Gutzwiller) in which sex and education are associated with preventive behaviour; and the physical environment, or the changes in health hazards or parts of the environment such as housing, pollution and noise.

An additional concern with data sources was seen in relation to the kinds of sources that have not been incorporated into the methodological debate on health promotion indicators. Reservations were expressed on the lack of use of public records other than official medical data. 
For example, the vast amount of information collected on business and economics in most western countries has not really been explored as a data source. Economic indicators are routinely used by countries to monitor the health of the economy. While these measures are fraught with a number of methodological difficulties, they often play a powerful role in decision-making that directly affects the health of populations. Another example, although very complex in interpretation, is the role of the media in conducting surveys and providing a surfeit of information on healthy lifestyles. It was felt that some health promotion indicators should take into account this powerful influence on public opinion.

Two major methodological strategies are widely used in data collection: the collection of routine, official, statistical information and the use of cross-sectional surveys, generally on specific target groups. The central problem with the use of routine data became obvious in the discussions on traditional health indicators: they are collected for purposes other than health promotion. Even so, this might not be an insurmountable problem. There are more damaging indictments of the use of such data. To begin with, this type of information is rarely timely; large-scale data collection agencies are ponderous institutions in most countries. They typically require years to publish information. The length of the delay of publication varies greatly from country to country, exacerbating the problem of comparability. In addition, the contextual problem, discussed above, becomes significant. Throughout the discussions of indicators, citizens of various countries constantly pointed to unique characteristics of their countries' data collection methods.

Cross-sectional surveys as a method for using health promotion indicators were widely discussed. Obviously many researchers, health educators and policy-makers are thoroughly familiar with this method; thus the issues and problems are keenly appreciated.

\section{Data analysis}

While much of the methodological debate on health promotion indicators research focuses on issues of measurement, there is increasing interest in problems of data analysis. This preoccupation with analysis cannot be separated from the fact that data analysis is undergoing serious recon- sideration within the social sciences in general. To a great extent, this re-evaluation is a result of the considerable growth in the past two decades of more powerful computers and the ready availability of complex analytical software (such as SPSS, SAS and Lisrel). In addition, the past decade has seen the rapid growth of personal computers, which have brought the technology to the researcher's desk. In brief, the researcher is now confronted with a bewildering array of analytic techniques, making very relevant the question of how this new technology may apply to indicator research. Even if there is general agreement on what indicators to use and how to measure them, analysis remains a formidable task.

Many of the papers in this issue of Health promotion are illustrative of these points. It has been argued that simple measures have many advantages: they are easily obtained; they are relatively easy to interpret; and they provide a semblance of comparability across countries. The likely result of such easily obtained measures, however, is a massive set of data that will present elaborate analytical problems. The end product, all too often, is an equally massive set of descriptive data. People working in health promotion are undoubtedly all too familiar with documents and reports with page after page of descriptive data that defy further interpretation. Usually, such materials can only be perused and then relegated to the bookshelf, to take their place with other weighty documents. Attempts to resurrect such data for secondary analysis are exceedingly time-consuming and often lead to results published years later.

Composite measures or indices of health promotion are a noble attempt to bypass some of the aforementioned analytic problems and several have been put forward in this issue of Health promotion. They offer a way out of some of the obvious problems associated with simple measures. Nevertheless, considerable pessimism has been expressed about their use. First, composite measures often contain items measured at different conceptual levels; that is, information collected from questionnaires given to individuals is mixed with information collected from records, or, within questionnaires, information obtained from an individual is mixed with an individual's reports on his or her social setting. Second, in the long run, composite measures may be considered reductionistic, tending artificially 
to reduce a multicomponent concept to a single number. These two difficulties are related to a third problem with composite indicators: weighting. Ideally, composite indicators should be developed and tested over a considerable time and on diverse populations in order to determine the strength of each of their components. Even appropriate testing may lead to the realization that there are no ideal weightings for the diversity of populations to which the measure might be applied. This, of course, compromises the comparability of such measures and again raises the thorny issue of the context in which the indicator is applied. Even the very best composite indicators of health status appear to require much context-specific development (see Kaplan's article). Finally, even if the weighting problem is satisfactorily resolved, the problem of interpreting the measure remains.

Whether simple or composite indicators are used, the ultimate analytical challenge is to use them in an appropriate statistical or mathematical procedure. From a methodological perspective, indicators are developed because they are markers in a broader system of causality. They fit into an implied scientific paradigm, that is, the expectation that, as the indicators fluctuate, other parts of a more complete system will be perturbed. An assumed underlying model drives the analysis. Certainly this notion of combining or linking indicators into a larger framework underlies the thinking of a large number of researchers who have been involved with indicators. This problem is explored further in the section on conceptual issues because it represents the very fine line between analytical and conceptual issues.

In reality, most indicators are not explored analytically to the extent that they could be. Thus, the literature, including this special issue, remains replete with simple descriptive statistics and elementary cross-tabulations. Clearly, these are first steps in analysis, but hardly represent the full use of available analytic possibilities. A principle failure is the general lack of techniques that employ some type of time-series analysis. This is a particular handicap in looking for indicators and analyses that are presumably sensitive to process and change.

The last methodological problem discussed here has been recognized as particularly salient for health promotion: how to use analytic techniques and methods that will make the meaning of the indicators clear to the end users. Data analysis has been a largely rarified endeavour, conducted by social scientists and often comprehensible to only a few. Thus, profound analysis of data, using the best of indicators, may be totally meaningless to the people who most need the results. Clearly, in all the discussions of health indicators this has been noted as a most severe problem. Fortunately it has been recognized as such and has been placed on the agenda for researchers.

\section{Concepts and theories}

The section on methodology repeatedly emphasized problems that carry us directly into conceptual and theoretical issues. Indeed, it is often difficult to distinguish whether the problem is conceptual or methodological and perhaps such a distinction is artificial. Nevertheless, discussion, workshops and writing during the past decade have ultimately locked on to the notion that a firm conceptual basis is the necessary first step to the solution of methodological problems. This has clearly been the experience in the Berne Workshop and all subsequent meetings. Although recognizably partitioning this discussion, the following section emphasizes the key role that concepts and theories take in the debate over the application and development of health promotion indicators.

\section{Defining the field}

An indicator is defined as a measure that summarizes information relevant to a particular phenomenon, or a reasonable proxy for such a measure. Two broad types of indicators can be distinguished: social indicators and research or scientific indicators. Social indicators are defined as measures that relate to social policy and decision-making, but there are many further elaborations of this definition. From a methodological point of view, indicators can be seen as surrogates for otherwise unmeasurable phenomena. This is a reasonably well accepted notion in the basic scientific paradigm underlying research: that is, a concept is not seen as something directly measurable, so an indicator or a marker is sought to show that the concept is really present. This philosophical position is usually translated into a more prosaic one in the search for indicators of health and health promotion.

Many attempts to define health promotion indicators, continued throughout the discussions 
of recent years, have turned to what indicators are for, because this may be seen as easier to answer than thorny epistemological questions. Health promotion indicators may be defined as being used for: health policy, health targets and expected policy results; health-related processes of planned programmes and activities; and health resources or policy inputs. In brief, many (such as Noack) argue that health promotion indicators reflect a general systems approach, concerned with the input, throughput and output of health promotion activities.

Within this perspective, health outcome measures appear to be most widely accepted and have probably the strongest implied conceptual base. For example, health status indicators have an appealing face validity and conceptual fit with an underlying biomedical paradigm. Further, they have intuitive appeal as final measures of interventions. Nevertheless, as pointed out earlier and in several other articles in this issue of Health promotion, they are generally measures of negative health, relying on standardized disease categories. Only a few of these types of measure can be seen to be directly relevant to health promotion (see Hunt's and Kaplan's articles). New developments in this area have argued for more scales of subjective health perceptions, which would tap latent dimensions of quality of life. These dimensions are often cited as: overall health, physical functioning, psychological or mental functioning, social functioning, social role behaviour, and role integration (Hunt et al., 1986; McDowell \& Newell, 1987).

There are many problems with the adaptation of these health status indicators to the field of health promotion. The most crucial is the lack of an underlying theory of health promotion. The underlying biomedical conceptualization of health status measures is assumed, but most critics would argue that this is not transferable to health promotion as a concept. An exact theory of health promotion has not yet been made.

Interest is growing in measuring and specifying the conditions or resources necessary for individual wellbeing. These conditions range from adequate shelter to a healthy physical environment. Groups of the regional targets for health for all (WHO, 1985) focus on lifestyle, the social environment, the health care system and social support systems. Within these groups, single targets address issues of central importance, such as: positive and negative health behaviour, coping, working conditions, primary and secondary health care, information systems and personal resources. However interesting this list, few adequate health promotion indicators can be identified and systematic efforts to specify the nature of appropriate indicators have failed to reach consensus.

In sum, the search for appropriate health promotion indicators has led to the unenviable task of defining a new conceptual field. Candidates from the general field of health indicators have been discussed at length without any resolution as to their applicability for health promotion. The realization has been that much work has to be done to find indicators appropriate to an emerging conceptual area. In general, WHO documents (Ottawa Charter for Health Promotion, 1986; WHO, 1985) have defined health promotion as including several key characteristics: the process of enabling people to increase control over and to improve their health, action to build a healthy public policy, the creation of supportive environments, the strengthening of community action for better health, the development of personal skills for health, and the reorientation of health care. One key principle underlies all of these: they are active concepts. Change is a main component of all six. The implication for indicator development is profound: any health promotion indicator should be capable of detecting movement.

In defining health promotion indicators to incorporate this emerging conceptualization, the debate turns inevitably to issues of methodology that have already been discussed. For example, should indicators try to make objective or subjective assessments of such notions as enablement and strengthening of community action? In a similar way, the issue of context again becomes relevant. But most significantly, the general underlying model of the natural sciences and its attendant implications become a central concern.

\section{Defining the theory}

As in other fields of social reporting, as well as in applied research, the development of health indicators has been very much influenced by pragmatic and methodological considerations. Theoretical aspects played a minor role, yet theoretical and paradigmatic issues can be assumed to have guided the development and use 
of health indicators, at least implicitly. There are at least three notable theoretical issues in the definition and/or selection of health promotion indicators: the role of the macro (broad social or environmental) perspective as opposed to a micro (individual) perspective; the notion of process as a property of health promotion, and the role of models and model building in articulating a theory.

The WHO regional indicators of health for all are designed to assess the attainment of the regional targets as defined within a broad framework, but are generally obtained at the individual level. In the long discussions of and acres of print about these targets and their indicators, little reference has ever been made to a theoretical perspective. This may provide some explanation for the seeming contradiction of specifying targets at a macro level while collecting information from a micro perspective. Much emphasis is placed on health-related behaviour, in which normality is assumed to be some way of acting at the individual level. Generally, models or theories have been principal means of obtaining conceptual clarity. It could be argued that appropriate models for health promotion in the social sciences might serve to bridge the problem of artificially dividing the world into individual and aggregate behaviour and phenomena.

If a model is to be valid then it should be isomorphic with some underlying theory (of which the model is a realization) and strong enough to withstand the same tests as the underlying theory. There is great risk in using models in health promotion, particularly to inform indicator development. One such risk is the attempted articulation of models before an underlying theory is conceptually clear. The model may have more than one possible theoretical interpretation (very common in the social sciences) or may appear useful but lack a theory. Several emergent principles need to inform any model for the development of health promotion indicators: contextualism, dynamism, and, probably, eclecticism.

Problems of theory are clearly central in discussions of issues and problems in health promotion indicators. The articles in this issue of Health promotion and the results of several meetings on indicators for health promotion make this apparent. Despite this growing awareness of the need for an articulated general theory of health promotion that would provide guide- lines for the selection and development of heaith promotion indicators, in general, little coordinated effort has been made to this end. Building a theory takes time, effort, research and a large amount of imagination and insight; none of these yields easily to any sense of urgency felt by those in the field of health promotion. One crucial factor is undoubtedly biographical. Most researchers and practitioners in health promotion have seen the field as one of action, not contemplation. This does not bode well for the development of a theory.

\section{Purpose and application}

The first column of Table 1 reveals some additional issues and problems associated with health promotion indicators. In preparing this article, we decided to discuss this column last because, in the many workshops, meetings and consultations on the topic, purpose and application always seemed to be at the end of the agenda. Nevertheless, we chose to put them in the first column of Table 1 to reflect their logical position in the development of indicators. After all, it is the consumer who is important.

Who wants these indicators and who is going to use them? In general, the answer has been: researchers and policy-makers. These groups are or have been very different. Most researchers have been based either in universities or research organizations; most policy-makers have been in government, politics or business. Thus, the expectations of users of health promotion indicators are very different at the outset. The meetings and efforts of the past decade have created an active dialogue between some representatives of these two factions and the people who have participated have moved into a position of empathy. Social scientists have become increasingly concerned about what happens to their research results and policy-makers have felt the need for better information. Thus a large number of issues and problems in research and policy have been identified.

\section{Research}

One of the major purposes of scientific research is undoubtedly to develop and test theories. During the last decade considerable progress has been made in social and epidemiological research linking sociological and behavioural variables with different health outcomes. This work has led to the development and application of a 
number of measures and some indicators of health resources, health-related processes and dimensions of health. For example, the Alameda County Study (Berkman \& Breslow, 1983) showed that indicators of social status, social support and lifestyle were independently and significantly correlated to rates of mortality and morbidity assessed during the nine years following the original collection of data. It seems that measures of positive health, however, have been included in such studies only very recently. Thus, testing theories on the maintenance and the improvement of health or hypotheses derived from such theories appears to be a major challenge for future work, which, of course, will require suitable measures and indicators.

It is therefore not surprising that hardly any work has been reported in which measures were developed to predict changes in health, to better the understanding of processes in health promotion and to develop and evaluate new methods and techniques in this field. Until very recently health promotion research has just not been an issue.

\section{Policy}

In several countries (Canada, France, Sweden and the United States) population surveys are being conducted at regular intervals to assess certain aspects of health and health care and to monitor changes. Within this tradition of policylinked or applied social science research, a relatively large number of measures and indicators have been developed of, for example, subjective health and ill health, and of health-enhancing and health-damaging behaviour, particularly so-called risk behaviour. As most of this work so far has been disease-oriented, however, the challenge will be to use these measures and to develop new ones within the wider framework of health promotion.

A second field in which health-related indicators have been extensively used is the evaluation of intervention programmes in the community and the workplace. The main focus of such programmes has been the reduction of cardiovascular risk factors and the prevention of premature death from or disability due to coronary heart disease. Thus, studies such as the North Karelia Project (Nissinen et al., 1987) included somatic risk factors, risk behaviour and, to a minor degree, some indicators of the progress of the intervention, such as the involve- ment of community organizations and of community physicians and nurses in the programme. Again, in order to move from a risk-factor approach to a health promotion strategy, these indicators may still be useful, but they will have to be interpreted in a different way. In addition, new measures will have to be developed that indicate what people actually do to increase their control over and to improve their health.

\section{CONCLUSIONS}

Over the last decade or so health promotion has emerged as a new field of activity and policy. As a new paradigm, health promotion has a wider perspective than the paradigm of disease prevention, medical care and medical treatment. Health promotion differs from prevention and care, however, in four distinct ways: in its orientation towards positive health, in its concern with the health of the whole population, in its emphasis on control of health, and health issues by the people and not by professionals and experts, and in that it is seen to be the key element of a new public health.

During this process the need for systematic scientific information on health and health promotion, including the need for health promotion indicators, has become apparent. Often high expectations are articulated, as if a system of reliable, valid and target-related health promotion indicators could solve many problems faced by a new, healthy public policy.

The process of developing indicators suggests that we must be more realistic. Health promotion indicators must be considered in the context of new healthy public policy and of health research. The following major problems need to be tackled and, we hope, resolved:

- planning, designing and carrying out health promotion research and developing useful qualitative categories and quantitative measures, including indicators;

- clarifying the needs for and implementing new health promotion policies and programmes, and developing and testing indicators related to specific aspects of such policies;

- developing and testing theories of health policy and health promotion processes that address the major health needs of populations, particularly the needs related to inequalities in health and the prevention of premature death and chronic disease. 
- specifying, within the context of such theories, the specific type of information needed on the social, economic, cultural and physical context of health promotion, and the social, psychological and biological processes involved;

- designing, developing and using measurement techniques that allow the tapping of key variables and concepts and the clarification of the limitations of measurement; and

- developing and testing methodologies for analysing data and presenting information so that the people involved can understand and use relevant health information, including new health promotion indicators.

\section{REFERENCES}

Abelin, T. et al., ed. (1987). Measurement in health promotion and protection. Copenhagen, WHO Regional Office for Europe (WHO Regional Publications, European Series, No. 22).
Anderson, R. et al. (in press). Health behaviour research and health promotion. Oxford, Oxford University Press.

Berkman, L.F. \& Breslow, L. (1983). Health and ways of living. New York, Oxford University Press.

Hunt, S. et al. (1986). Measuring health status. London, Croom Helm.

McDowell, I. \& Newell, C. (1987). Measuring health: a guide to rating scales and questionnaires. New York, Oxford University Press.

Nissinen, A. et al. (1987). Experiences from the North Karelia Project. In: Abelin, T. et al., ed. Measurement in health promotion and protection. Copenhagen, WHO Regional Office for Europe (WHO Regional Publications, European Series, No. 22), pp. 414-429.

Ottawa Charter for Health Promotion (1986). Health promotion, 1(4): iii-v.

WHO (1984). Health promotion. A discussion document on the concept and principles of health promotion. Copenhagen, WHO Regional Office for Europe (unpublished document ICP/HSR 602 (m01)).

WHO (1985). Targets for health for all. Copenhagen, WHO Regional Office for Europe.

WHO (1986). Workshop on Measuring Health Behaviour and Health: towards New Health Promotion Indicators. Berne, 15-18 June. Copenhagen, WHO Regional Office for Europe (unpublished document ICP/HSR 648 (S)). 
